OPEN ACCESS

Edited by:

Diana Santelia,

ETH Zürich, Switzerland

Reviewed by:

Ashwani Pareek,

Jawaharlal Nehru University, India

Marcelo Nogueira Do Amaral,

Federal University of Pelotas, Brazil

*Correspondence:

Chunwu Yang

yangcw809@nenu.edu.cn

Specialty section: This article was submitted to

Plant Abiotic Stress,

a section of the journal

Frontiers in Plant Science

Received: 03 January 2021

Accepted: 06 April 2021

Published: 22 April 2021

Citation:

Guo R, Zhao L, Zhang K, Lu H, Bhanbhro N and Yang C (2021)

Comparative Genomics and Transcriptomics of the Extreme Halophyte Puccinellia tenuiflora

Provides Insights Into Salinity Tolerance Differentiation Between

Halophytes and Glycophytes.

Front. Plant Sci. 12:649001.

doi: $10.3389 /$ fpls.2021.649001

\section{Comparative Genomics and} Transcriptomics of the Extreme Halophyte Puccinellia tenuiflora Provides Insights Into Salinity Tolerance Differentiation Between Halophytes and Glycophytes

\author{
Rui Guo', Long Zhao ${ }^{2,3}$, Kaijian Zhang ${ }^{4}$, Huiying Lu$^{2}$, Nadeem Bhanbhro ${ }^{2}$ and \\ Chunwu Yang ${ }^{2 *}$
}

${ }^{1}$ Key Laboratory of Dryland Agriculture, Institute of Environment and Sustainable Development in Agriculture, Chinese Academy of Agricultural Sciences, Beijing, China, ${ }^{2}$ Key Laboratory of Molecular Epigenetics of Ministry of Education (MOE), Northeast Normal University, Changchun, China, ${ }^{3}$ Institute of Genetics and Developmental Biology, Chinese Academy of Sciences, Beijing, China, ${ }^{4}$ Beijing Novogene Bioinformatics Technology Ltd., Beijing, China

Halophytes and glycophytes exhibit clear differences in their tolerance to high levels of salinity. The genetic mechanisms underlying this differentiation, however, remain unclear. To unveil these mechanisms, we surveyed the evolution of salinity-tolerant gene families through comparative genomic analyses between the model halophyte Puccinellia tenuiflora and glycophytic Gramineae plants, and compared their transcriptional and physiological responses to salinity stress. Under salinity stress, the $\mathrm{K}^{+}$concentration in the root was slightly enhanced in P. tenuiflora, but it was greatly reduced in the glycophytic Gramineae plants, which provided a physiological explanation for differences in salinity tolerance between $P$. tenuiflora and these glycophytes. Interestingly, several $\mathrm{K}^{+}$uptake gene families from $P$. tenuiflora experienced family expansion and positive selection during evolutionary history. This gene family expansion and the elevated expression of $\mathrm{K}^{+}$uptake genes accelerated $\mathrm{K}^{+}$accumulation and decreased $\mathrm{Na}^{+}$ toxicity in $P$. tenuiflora roots under salinity stress. Positively selected $P$. tenuiflora $\mathrm{K}^{+}$ uptake genes may have evolved new functions that contributed to development of P. tenuiflora salinity tolerance. In addition, the expansion of the gene families involved in pentose phosphate pathway, sucrose biosynthesis, and flavonoid biosynthesis assisted the adaptation of $P$. tenuiflora to survival under high salinity conditions.

Keywords: gene family expansion, genome, halophyte, Puccinellia tenuiflora, RNA-seq, positive selection

Abbreviations: ABA, abscisic acid; $\mathrm{NHX}, \mathrm{Na}^{+} / \mathrm{H}^{+}$exchanger; PPP, pentose phosphate pathway; DEG, differentially expressed gene; NCED, 9-cis-epoxycarotenoid dioxygenase; ABF, abscisic acid-responsive element binding protein; HAK, KUP/HAK/KT K $\mathrm{K}^{+}$transporter; $\mathrm{V}-\mathrm{H}^{+}$-ATPase, vacuolar $\mathrm{H}^{+}$-ATPase; HKT, high-affinity $\mathrm{K}^{+}$transporter; AKT, low-affinity $\mathrm{K}^{+}$transporter; 6PGDH, 6-phosphogluconate dehydrogenase; LEA, late embryogenesis abundant protein. 


\section{HIGHLIGHTS}

- The evolution of salinity-tolerance genes has contributed to the development of salinity tolerance in the extreme halophyte Puccinellia tenuiflora.

- Root potassium concentration was slightly enhanced in $P$. tenuiflora under salinity stress, but it was greatly reduced in glycophytic Gramineae plants.

- Several potassium channel and potassium transporter gene families from $P$. tenuiflora were expanded and positively selected during the species' evolutionary history.

\section{INTRODUCTION}

Soil salinization is a severe environmental problem that limits agricultural production (Flowers and Yeo, 1995; Flowers et al., 2010). Saline soil contains various concentrations and types of salts, and soil salinity levels can reach $2,380 \mathrm{mM} \mathrm{Na}^{+}$. Saltaffected soils may contain $\mathrm{NaCl}$ alone, $\mathrm{NaCl}$ and $\mathrm{Na}_{2} \mathrm{SO}_{4}$, or $\mathrm{NaCl}$ and $\mathrm{Na}_{2} \mathrm{CO}_{3}$. Plant growth under saline conditions is influenced by multiple stress factors, including direct ion injury and osmotic stress inflicted by $\mathrm{Na}^{+}$and $\mathrm{Cl}^{-}$and indirect nutrient stresses caused by competition from $\mathrm{Na}^{+}$and $\mathrm{Cl}^{-}$(Munns and Tester, 2008). Tolerance to salinity stress is imparted by a complex network that affects almost all plant metabolic and developmental processes that are controlled by numerous genes. Higher plants can be classified as halophytes or glycophytes according to their ability to tolerate salinity stress. Halophytes can survive and spend their entire life cycle at $\geq 200 \mathrm{mM} \mathrm{NaCl}$ (Flowers et al., 2010). Many halophytes can even survive conditions of $>1000 \mathrm{mM}$ salinity, whereas most glycophytes can only survive under conditions of 50-100 mM salinity (Munns and Tester, 2008). The molecular study of salinity tolerance has largely focused on model glycophytes, such as rice and Arabidopsis (Munns and Tester, 2008; Suzuki et al., 2016; Wang and Xia, 2018; Ganie et al., 2019; Wang et al., 2019; Zhu et al., 2019), however, relatively few attentions have been paid to the molecular mechanisms underlying salinity tolerance in halophytes.

Although halophytes and glycophytes exhibit dramatic differences in salinity tolerance, they share most of the characteristics necessary for survival in saline soils. These include the control or excretion of $\mathrm{Na}^{+}$and $\mathrm{Cl}^{-}$in the root, compartmentalization of toxic ions in organs or cells, synthesis of compatible organic solutes in the cytoplasm, and maintenance of sufficient concentrations of key nutrients such as $\mathrm{K}$ and $\mathrm{N}$ (Flowers and Colmer, 2008; Wang and Xia, 2018). Halophytes have evolved a strong tolerance to salinity over history and the role played by genome evolution in establishing differences in salinity tolerance between halophytes and glycophytes is of considerable interest.

Puccinellia tenuiflora $(2 n=14)$ is a forage grass with high nutritional value and strong tolerance for salinity and alkaline stresses (Yan and Sun, 2000; Zhao et al., 2011; Meng et al., 2016). This species is even able to grow normally under extreme conditions (e.g., salinity $>600 \mathrm{mM}$ and $\mathrm{pH} 10-11$ ) ( $\mathrm{Xu}, 1990$; Xu et al., 1995; Wang et al., 2006; Zhang et al., 2013). Many molecular studies of salinity-tolerance have focused on this halophyte (Wang et al., 2007a,b; Liu et al., 2009; Ardie et al., 2009, 2010, 2011; Yu et al., 2011; Zhang et al., 2013, 2017; Yu et al., 2013). To reveal the regulation of gene expression and genomic mechanisms underlying the differences in salinity tolerance between halophytes and glycophytes, we conducted comparative genomic and transcriptome analyses between $P$. tenuiflora and several glycophytic Gramineae plants. Our results improved the current understanding of salinity tolerance in halophytes and provided insights into the mechanisms underlying differences between halophytes and glycophytes.

\section{MATERIALS AND METHODS}

\section{Plant Materials and Reference Genome}

The taxonomy characteristics of Puccinellia tenuiflora are available at Flora of $\left(\mathrm{China}^{1}\right)$. P. tenuiflora was bred as a grass variety and is not an endangered or protected species, so no specific permissions were required for its application in this study. The reference genome of $P$. tenuiflora used in this study was reported in our previous publication (Guo et al., 2020). The genome of Dactylis glomerata was reported in Huang et al. (2020). The reference genome with integrated annotation files of D. glomerata is publicly available at https: //orchardgrassgenome.sicau.edu.cn/. In the current study, we used the P. tenuiflora variety 'Baicheng' for RNA sequencing (RNAseq) and physiological measurements. The P. tenuiflora seeds were provided by Jilin Academy of Agricultural Sciences, Changchun, China.

\section{Gene Family Discovery}

Gene families were discovered using OrthoMCL software ${ }^{2}$ (Li et al., 2003). The whole genome sequences, protein sequences, and transcript sequences of 16 species (Thellungiella salsuginea, Arabidopsis thaliana, Phalaenopsis equestris, Elaeis guineensis, D. glomerata, Musa acuminata, Oryza sativa, Brachypodium distachyon, Setaria italica, Zea mays, Hordeum vulgare, Aegilops tauschii, Sorghum bicolor, Ananas comosus, Zostera marina, and Amborella trichopoda) were obtained from NCBI, Ensembl, and http://marinegenomics.oist.jp/gallery/. To exclude putative fragmented genes, the genes encoding protein sequences shorter than 50 amino acids were filtered (Yuan et al., 2018). The longest transcript was selected if a gene showed alternative splicing. Finally, gene family clustering was carried out using OrthoMCL software with a 1.5 inflation index (Li et al., 2003). We used InterProscan software and the Pfam motifs of Late Embryogenesis Abundant (LEA)1, LEA2, LEA3, LEA4, LEA5, DEHYDRIN, and Seed Maturation Protein (SMP) to discover the LEA superfamily members present in the genomes of P. tenuiflora, O. sativa, $H$. vulgare, A. tauschii, B. distachyon, and D. glomerata (Finn et al., 2017). We constructed the polygenetic tree of the LEA5 family using the MEGA-X-based NeighborJoining method (Kumar et al., 2018).

\footnotetext{
${ }^{1}$ http://www.efloras.org/florataxon.aspx?flora_id=2\&taxon_id=200026128

${ }^{2}$ https://orthomcl.org/orthomcl/app
} 


\section{Phylogenetic Tree and Divergence Time}

We applied the sequences of 228 single copy orthologs to generate the phylogenetic tree. We aligned the protein sequences of these orthologs using MUSCLE software (Edgar, 2004), and then the protein alignments were translated to CDS sequences. The phylogenetic tree was generated using RAxML (for maximum likelihood; 7.2.3) (Stamatakis, 2006; Stamatakis et al., 2008). The MCMCTREE model of PAML was selected to calculate the divergence time between $P$. tenuiflora and the other 16 species ( main parameters: burn-in $=10,000$, sample-number $=100,000$, sample-frequency $=2$, and clock $=2$ ) (Yang, 2007). Three calibration points used were based on the study by Cai et al. (2015) and the TimeTree website ${ }^{3}$.

\section{Expansion and Contraction of Gene Families and Positive Selection Analysis}

Genes from different species were clustered into gene families using OrthoMCL software (see Text Footnote 3) with a 1.5 inflation index (Li et al., 2003). The gene families were excluded from the analysis if the gene number was $\geq 200$ in one species or $\leq 2$ in all other species. The Cafe software package was applied to determine the expansion and contraction of gene families (De Bie et al., 2006; Han et al., 2013). Gene gains and losses in gene families in a phylogeny were tested by calculating the $P$-values of each branch using the Viterbi method with a randomly generated likelihood distribution. The exact $P$-values for transitions between the parent and child family sizes were estimated using this method for all branches of the phylogenetic tree (De Bie et al., 2006; Han et al., 2013). Positive selection analysis was based on the alignments of protein sequences. We used MUSCLE software to align the protein sequences of single copy genes in P. tenuiflora, O. sativa, $H$. vulgare, and A. tauschii (Edgar, 2004) and Gblocks to filter poor positions and transform protein alignments to CDS (Castresana, 2000). Positive selection sites were discovered with the branch-site model (Zhang et al., 2005) of PAML (Yang, 2007) by setting P. tenuiflora as the foreground branch. The $P$-values were generated using the $\chi^{2}$ test, and then adjusted by the false discovery rate (FDR) method.

\section{LTR Retrotransposons Insert Time Assessment}

Nucleotide variations at the $5^{\prime}$ and $3^{\prime}$ ends of intact LTR retrotransposons and DNA substitution rates were calculated (Huang et al., 2020). The nucleotide variations and DNA substitution rates were used to estimate the insert time of LTR retrotransposons according to the method described by Ma and Bennet-zen (2004).

\section{Motif Analysis}

We carried out a motif analysis of the low affinity $\mathrm{K}^{+}$ transporter (AKT), $\mathrm{Na}^{+} / \mathrm{H}^{+}$exchanger (NHX), and high-affinity $\mathrm{K}^{+}$transporter (HKT) proteins among O. sativa, H. vulgare, A. tauschii, $P$. tenuiflora, and D. glomerata using MEME Suite

${ }^{3} \mathrm{http}: / /$ www.timetree.org
5.2 (Bailey et al., 2009). The orthologous genes of AKT, HKT, and NHX for each species were identified by the best BLASTn hit against the corresponding rice sequence (qcovs $\geq 0.5, E$-value $\leq-7$, and identity $\geq 60 \%$ ).

\section{Comparative Transcriptome and Physiological Measurement of P. tenuiflora and Glycophytic Gramineae Plants}

Seeds of $P$. tenuiflora (variety Baicheng), O. sativa (variety JIJING88), H. vulgare (variety Zhu4300), D. glomerata (variety Sparta), and Triticum aestivum (variety Chinese Spring) were planted in plastic pots containing washed sand under greenhouse conditions $\left(24-26^{\circ} \mathrm{C}\right.$ day and $18-21^{\circ} \mathrm{C}$ night under 16 -h light). The rice and wheat seeds were provided by Dr. Bao Liu (Northeast Normal University, Changchun, China), and the $H$. vulgare seeds and D. glomerata seeds were provided by the Chinese Academy of Agricultural Sciences (Beijing, China). The pots (15 plants per pot) were cultured for 15 days with halfstrength Hoagland nutrient solution. We stressed 15-day-old seedlings (at about the three leaves stage) through daily exposure to half-strength Hoagland nutrient solution containing $300 \mathrm{mM}$ $\mathrm{NaCl}$. Control plants were watered with half-strength Hoagland nutrient solution. We used a randomized complete block design. After 12 and $48 \mathrm{~h}$ of exposure to salinity stress, we collected physiological measurement samples from all of the five species and RNA samples from $P$. tenuiflora and $D$. glomerata. There were three biological replicates of each treatment and tissue, and each biological replicate comprised a pool of 5-20 plants. A total of $2 \mu \mathrm{g}$ RNA per sample was used as the input material for the RNA sample preparations. Sequencing libraries were generated using a NEBNext UltraTM RNA Library Prep Kit for Illumina (NEB, United States) following manufacturer's recommendations and index codes were added to attribute sequences to each sample. All downstream analyses were performed with high quality clean data. About $6 \mathrm{~Gb}$ of clean data for each sample were used to perform the transcriptional analysis. Statistically significant differentially expressed genes (DEGs) were discovered using the DESeq2 $\mathrm{R}$ package, and DEG was defined by the adjusted $P$-value (FDR) $<0.05$ and Fold Change $\geq 2$. The DEGs were subjected to gene ontology (GO) and Kyoto Encyclopedia of Genes and Genomes (KEGG) enrichment analyses with a GOseq R package (Young et al., 2010) and KOBAS software (Mao et al., 2005), respectively. We discovered ortholog of each $P$. tenuiflora gene in the D. glomerata genome using the best BLAST hit based on the CDS sequence (bit score $\geq 200, E$-value $\leq-7$, and identified $\geq 60 \%$ ). Quantitative real-time PCR (qRTPCR) was employed to validate the results of the transcriptome analysis. RNA samples from the $P$. tenuiflora roots taken at $48 \mathrm{~h}$ after the onset of salt treatment were treated with DNaseI (Invitrogen), reverse-transcribed using SuperScript ${ }^{\mathrm{TM}}$ RNase HReverse Transcriptase (Invitrogen), and then subjected to qRTPCR analysis. Amplification of the target genes was monitored by SYBR Green. Actin and GAPDH were used as internal control genes. The gene expression data were analyzed using the 
$\Delta \Delta C$ t method (Livak and Schmittgen, 2001). Dried samples of roots and shoots were digested in $65 \% \mathrm{HNO}_{3}$ at $120^{\circ} \mathrm{C}$, and their $\mathrm{Na}^{+}$and $\mathrm{K}^{+}$contents were measured using an atomic absorption spectrophotometer (TAS-990super, PERSEE, China). The statistical analysis ( $t$-test) of the qRT-PCR and physiological measurements was performed using SPSS 16.0 (SPSS, Chicago, IL, United States).

\section{RESULTS}

\section{Gene Family Analysis}

The point of divergence between $P$. tenuiflora and D. glomerata was about 12.8 million years ago (Figure 1A). A total of 1, 233 gene families were unique to $P$. tenuiflora and potentially related to the strong salinity tolerance found in this species (Figure 1B). The gene number of $P$. tenuiflora was similar to that of O. sativa, Z. mays, D. glomerata, and S. italica (Supplementary Figure 1). The genome of $P$. tenuiflora contained $691 \mathrm{Mb}$ of transposable elements (62.44\% of the total sequence), including $580 \mathrm{Mb}$ of LTR retrotransposons (52.43\%) (Guo et al., 2020). We estimated the insertion time for the LTRs in $P$. tenuiflora, O. sativa, D. glomerata, $H$. vulgare, A. tauschii, and B. distachyon (Figure 1C). The LTR insertion peak indicates that a frequent insertion event occurred. The LTR insertion peak of $P$. tenuiflora was between those of O. sativa and D. glomerata (Figure 1C). The frequent LTR insertion events for $P$. tenuiflora occurred much later than those of its close relative, $H$. vulgare. We applied OrthoMCL software and Cafe software to conduct the expansion and contraction analysis of the gene family and members of all of the expanded $P$. tenuiflora gene families are listed in Supplementary Table 1. We conducted GO and KEGG enrichment analyses for all of the expanded genes listed in Supplementary Table 1. The GO enrichment analysis indicated that the expanded $P$. tenuiflora genes were enriched in many metabolic processes such as ion binding, sucrose synthase, G-protein activated inward rectifier potassium channel activity, organic substance metabolic process, and primary metabolic process (Supplementary Table 2 and Figure 2A). The KEGG enrichment showed that the expanded $P$. tenuiflora genes were enriched in the plant-pathogen interaction pathway, pentose phosphate pathway (PPP), phenylalanine metabolism, flavone and flavonol biosynthesis, flavonoid biosynthesis, and biosynthesis of amino acids (Figure 2B). We were particularly interested in expanded salinity-tolerance gene families and found that the inward rectifier potassium channel, voltagegated potassium channel, potassium transporter, flavonoid $3^{\prime}, 5^{\prime}$ hydroxylase, chalcone synthase, and sucrose synthase gene families of $P$. tenuiflora were expanded (Figure 2C and Supplementary Table 1).

Positive selection analysis revealed that 1,324 P. tenuiflora genes were positively selected over time (Supplementary Table 3). These genes were enriched in only eight GO terms, including the response to abiotic stimulus, response to temperature stimulus, response to freezing, and response to stress (Figure 3). Many salinity-tolerant genes of $P$. tenuiflora were found also to have been positively selected during evolutionary history, including six voltage-gated potassium channel genes, two inward rectifier potassium channel genes, and one potassium channel gene (Table 1).

\section{Evolutionary History of Salinity-Tolerance Families}

Here we focused on the evolution of several critical salinity tolerance gene families including the HKT, AKT, NHX, and LEA families (Figure 4 and Supplementary Figures 2-4). The LEA superfamily is divided into seven subfamilies on the basis of their distinctive motifs: LEA1, LEA2, LEA3, LEA4, LEA5, SMP, and DEHYDRIN. The member number in the LEA5 subfamily of $P$. tenuiflora was much higher than that of D. glomerata, O. sativa, $H$. vulgare, B. distachyon, and A. tauschii (Figure 4A). Although the gene number in $P$. tenuiflora was much lower than that in $H$. vulgare and A. tauschii (Supplementary Figure 1), the LEA5 number was much higher in $P$. tenuiflora than in $H$. vulgare and $A$. tauschii (Figure 4A). We generated a phylogenetic tree of the LEA5 families of $P$. tenuiflora, $O$. sativa, $H$. vulgare, $B$. distachyon, A. tauschii, and D. glomerata. The phylogenetic tree illustrated that the LEA5 family of $P$. tenuiflora expanded via duplication (Figure 4B). We conducted a comparative protein motif analysis of $P$. tenuiflora and several glycophytic Gramineae plants for $A K T, H K T$, and $N H X$ genes and found that $P$. tenuiflora and the glycophytic Gramineae plants displayed similar protein motif components for all of the selected genes (Supplementary Figures 2-4).

\section{Ion Content}

In the shoots of all of the four glycophytic Gramineae plants, the $\mathrm{Na}^{+}$concentration was greatly enhanced under salinity stress at both the 12 and $48 \mathrm{~h}$ time points (Figure 5A). However, the shoot $\mathrm{Na}^{+}$concentration in P. tenuiflora was unaffected at $12 \mathrm{~h}$ and slightly enhanced at $48 \mathrm{~h}$ under salinity stress (Figure 5A). Under salinity stress, $P$. tenuiflora and the glycophytic Gramineae plants displayed similar levels of $\mathrm{Na}^{+}$in their roots (Figure 5A). The $\mathrm{K}^{+}$accumulation in the shoots was unaffected in all of the four glycophytic Gramineae plants at both 12 and $48 \mathrm{~h}$ but enhanced in $P$. tenuiflora at $48 \mathrm{~h}$ (Figure 5B). When the seedlings were exposed to salinity stress for $12 \mathrm{~h}$, the root $\mathrm{K}^{+}$concentration decreased in D. glomerata and $T$. aestivum but not in $H$. vulgare, O. sativa, or P. tenuiflora. However, when the seedlings were exposed to salinity stress for $48 \mathrm{~h}$, the concentration of $\mathrm{K}^{+}$in the roots significantly decreased in all of the four glycophytic Gramineae plants, while being elevated by $49.5 \%$ in $P$. tenuiflora (Figure 5B). Under salinity stress, the $\mathrm{Na}^{+} / \mathrm{K}^{+}$ratio was much lower in $P$. tenuiflora than in the four glycophytic Gramineae plants in both shoots and roots at $48 \mathrm{~h}$ (Supplementary Figure 5).

\section{Transcriptional Profiling of $\boldsymbol{P}$. tenuiflora Under Salinity Stress}

When the seedlings were exposed to salinity stress for $12 \mathrm{~h}$, 1,628 DEGs were present in the roots and 760 in the leaves of $P$. tenuiflora. When the seedlings were exposed to salinity stress for $48 \mathrm{~h}, 2,068$ DEGs were present in the 


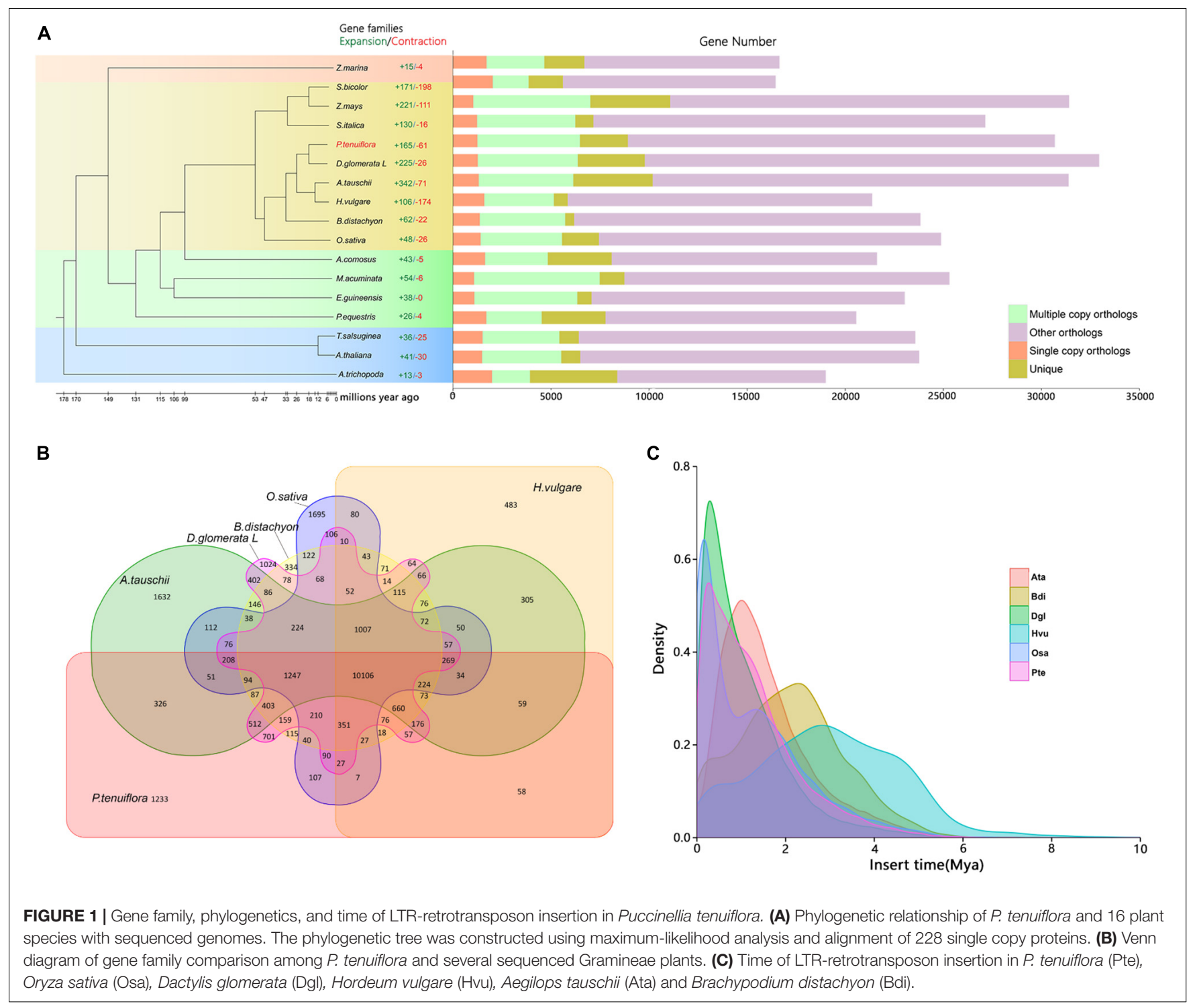

roots and 1,109 in the leaves of $P$. tenuiflora (Supplementary Table 4). We subjected the DEGs to KEGG enrichment analyses (Supplementary Figure 6), specifically focusing on the DEGs involved in $\mathrm{K}^{+} / \mathrm{Na}^{+}$homeostasis and osmotic adjustment (Supplementary Table 5). In the roots of P. tenuiflora, a HAK (KUP/HAK/KT $\mathrm{K}^{+}$transporter) gene (ID: PtO_2103.26) and HKT1:3 gene (ID: PtO_1129.21) were upregulated at $48 \mathrm{~h}$, and two voltage-gated potassium channel genes (IDs: PtO_1129.2 and PtF_428.24) were upregulated at both 12 and $48 \mathrm{~h}$ (Supplementary Table 5). Two $\mathrm{NHX}\left(\mathrm{Na}^{+} / \mathrm{H}^{+}\right.$exchanger) genes and a $\mathrm{V}-\mathrm{H}^{+}$- ATPase gene were upregulated in the roots of P. tenuiflora at $12 \mathrm{~h}$ or $48 \mathrm{~h}$ but not in the leaves (Supplementary Table 5). Twenty-nine LEA genes and 12 dehydrin genes were upregulated in the roots or leaves of P. tenuiflora (Supplementary Table 5). Glucose-6-phosphate dehydrogenase (G6PDH) is the rate-controlling gene for the PPP, sucrose synthase genes are central to sucrose biosynthesis, and flavonoid 3',5'-hydroxylase genes are critical to flavonoid biosynthesis. Two flavonoid 3',5'-hydroxylase genes (IDs: PtF_517.39 and PtO_820.20) were upregulated in the leaves of $P$. tenuiflora at $12 \mathrm{~h}$, and $G 6 P D H$ (PtO_647.1) was upregulated in the roots of P. tenuiflora at $12 \mathrm{~h}$ (Supplementary Table 5). Three sucrose synthase genes (IDs: PtO_1407.1, PtO_1927.24, and PtO_999.27) were upregulated in the roots or leaves of $P$. tenuiflora. Five abscisic acid ( $A B A)$ responsive element binding factor $(A B F)$ genes, which are core ABA signaling genes and two 9-cis-epoxycarotenoid dioxygenase (NCED) genes, which are critical to ABA biosynthesis, were upregulated in the roots or leaves of $P$. tenuiflora. Some superoxide dismutase genes and glutathione S-transferase genes were also upregulated in P. tenuiflora. The qRT-PCR experiment was carried out using gene-specific primers to validate the results of the RNAseq experiment (Supplementary Table 6). For eight of the 11 genes tested, the fold-change values from the RNAseq were similar to those from the qRT-PCR data, indicating that the results from the RNAseq were reliable (Supplementary Table 6). 


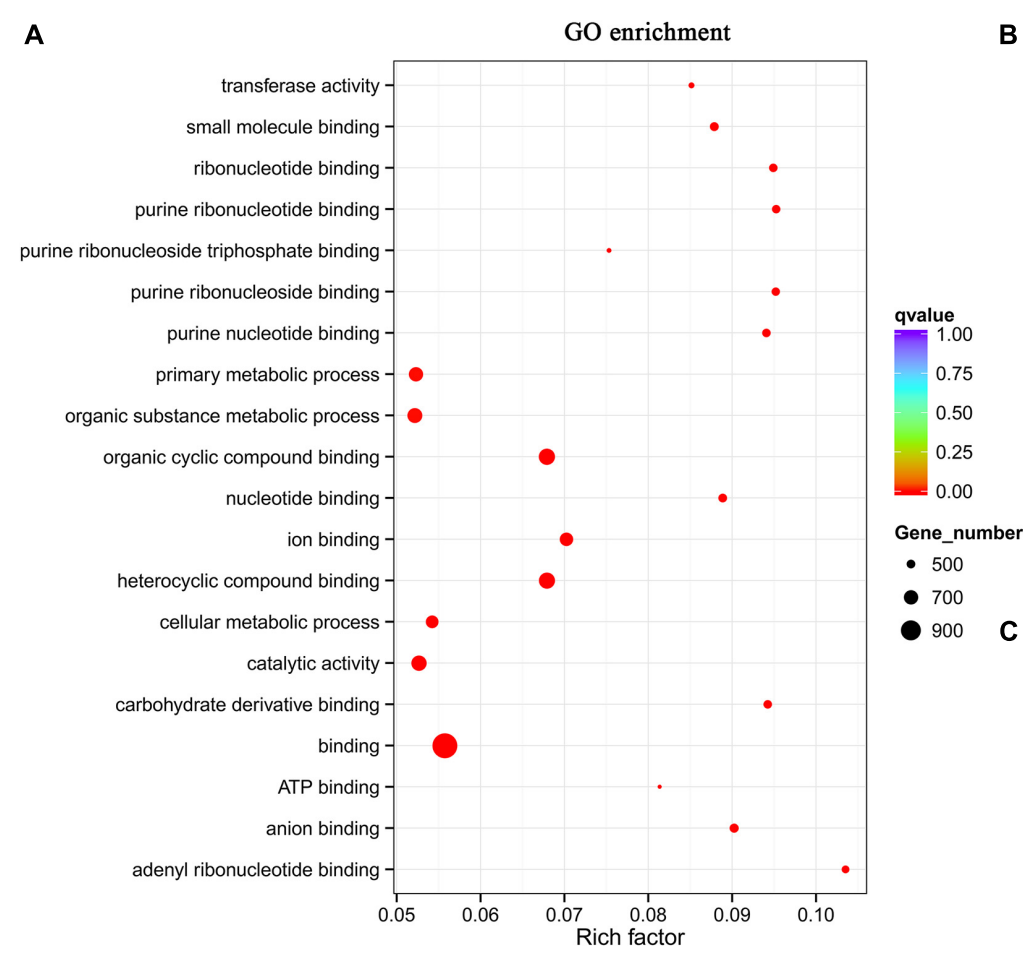

B

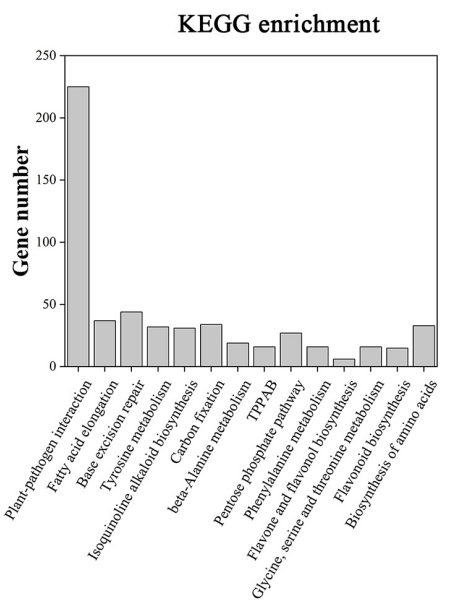

C

FIGURE 2 | Gene family expansion analysis of $P$. tenuiflora during evolutionary history. Gene family expansion analysis was conducted using OrthoMCL and Cafe software. (A) GO enrichment of $P$. tenuiflora gene families that expanded during evolutionary history. Only the top 20 enriched GO terms are displayed. (B) KEGG enrichment of the $P$. tenuiflora gene families that expanded during evolutionary history. Only the enriched KEGG pathways with adjusted $P$-values $<0.05$ are displayed. (C) Expanded salinity-tolerant gene families of $P$. tenuiflora during evolutionary history.

\section{Comparative Orthologous Expression Response of $P$. tenuiflora and D. glomerata}

To compare the gene expression response of $P$. tenuiflora and D. glomerata to salinity stress, we identified the ortholog of each $P$. tenuiflora gene in the D. glomerata genome using the BLAST program. We displayed the gene expression difference between $P$. tenuiflora and $D$. glomerata in a rectangular coordinate system with $x=\log 2$ (fold change of the $P$. tenuiflora ortholog) and $y=\log 2$ (fold change of the D. glomerata ortholog) for each gene; the fold change was the ratio of stress to control of the expression level (Figure 6A). About 60\% of the expressed genes were present in quadrant I and quadrant III, in which the P. tenuiflora ortholog ( $\mathrm{PtO})$ and D. glomerata ortholog (DgO) displayed similar responses to salinity stress (Figure 6A). We also compared the expression response of the $\mathrm{PtO}$ and $\mathrm{DgO}$ to salinity stress for each salinity-tolerance gene (Figure 6B). For several of the LEA genes, the PtO and DgO were upregulated either in the roots or leaves. Both the $\mathrm{PtO}$ and $\mathrm{DgO}$ showed upregulation of five dehydrin genes in roots at both 12 and $48 \mathrm{~h}$. The PtO and $\mathrm{DgO}$ of a flavonoid $3^{\prime}, 5^{\prime}$-hydroxylase gene were upregulated in leaves at $12 \mathrm{~h}$. G6PDH was upregulated in roots of $P$. tenuiflora but not in roots of D. glomerata at $12 \mathrm{~h}$. Both the PtO and DgO of NCED gene were upregulated in leaves at 12 and $48 \mathrm{~h}$ and in roots at $12 \mathrm{~h}$ (Figure 6B). The $\mathrm{PtO}$ of the $A B F$ gene was upregulated in leaves at $48 \mathrm{~h}$ and that of the $\mathrm{DgO}$ was upregulated in both leaves and roots at $12 \mathrm{~h}$ (Figure 6B). HKT1;3 was upregulated in the roots of $P$. tenuiflora but not in the roots of $D$. glomerata at $48 \mathrm{~h}$ (Figure 6B). The potassium channel KAT gene (KAT), $V$ $\mathrm{H}^{+}$-ATPase gene, and NHX gene were upregulated in the roots of P. tenuiflora at both 12 and 48 h but not in roots of $D$. glomerata. In summary, $\mathrm{PtO}$ and $\mathrm{DgO}$ exhibit similar expression responses to salinity stress in the ABA pathway, dehydrin, and LEA genes, but they displayed different expression responses in $H K T 1 ; 3$, $V$ - $H^{+}$-ATPase, NHX, and G6PDH.

\section{DISCUSSION}

\section{Comparative Genomics}

The comparative genomics results showed that the frequent LTR insertion event of $P$. tenuiflora occurred later than that of its close species, $H$. vulgare and D. glomerata. The LTR insertion events in P. tenuiflora occurred mostly during the Pleistocene Epoch, which lasted from approximately 2.6 million years to 11,700 years ago, including at least five documented major ice ages. Repeated ice ages (glacial cycles) can result in frequent change in sea-level due to the exchange of water between ice and ocean (Lambeck et al., 2014). The circular change of sea level may increase land salinity, which may be the main factor for the formation of extant saline land. During this period, the LTR of $P$. tenuiflora was frequently activated, which led to the rapid reorganization of its 


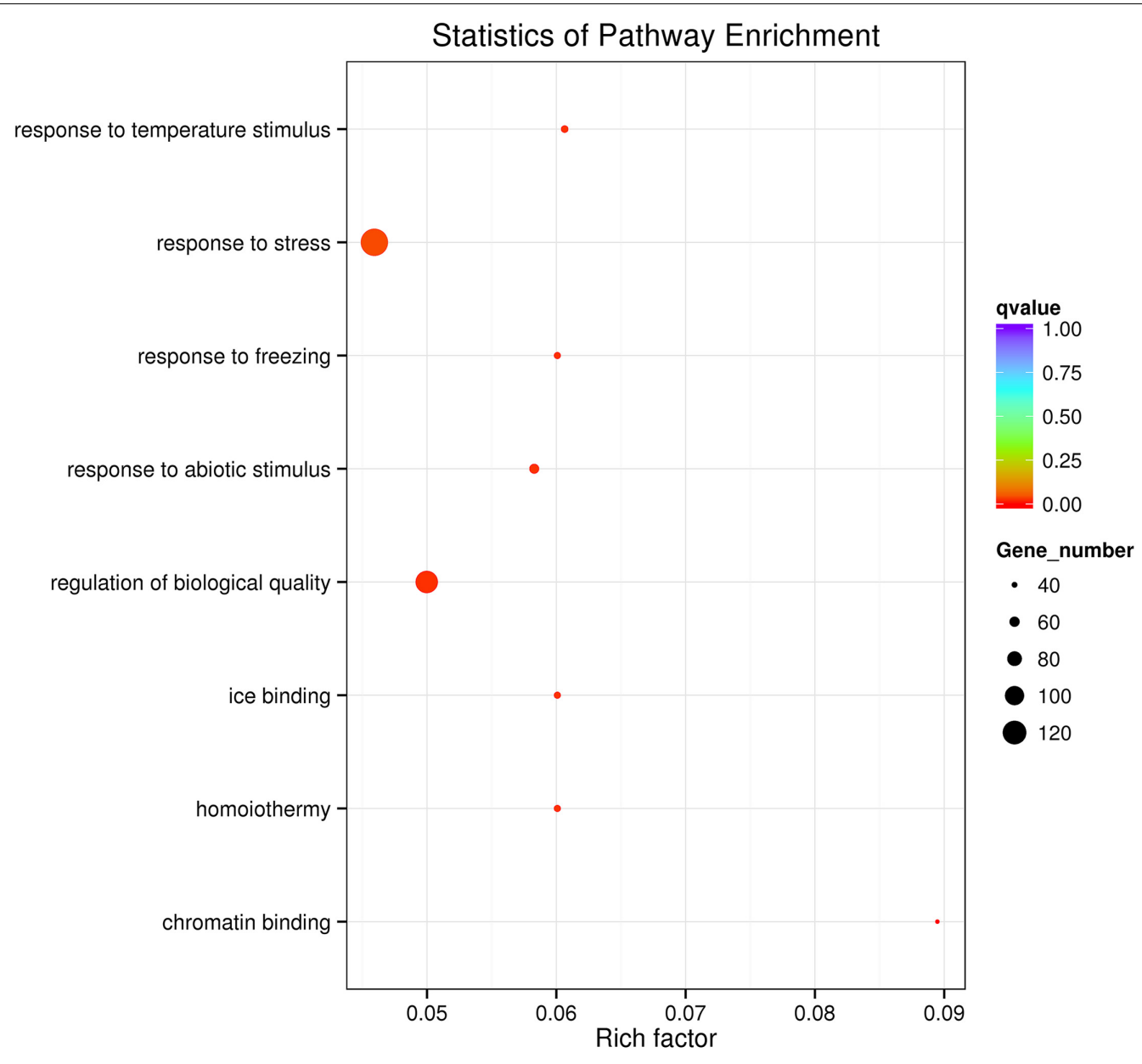

FIGURE 3 | Gene ontology enrichment of $P$. tenuiflora genes that were positively selected during evolutionary history.

genomes to adapt to increasing soil salinity. P. tenuiflora also has very strong resistance to chilling stress (Zhao et al., 2011, 2016). The frequent LTR insertion events in P. tenuiflora during the Pleistocene Epoch were likely to have contributed to the development of its strong tolerance to cold and multiple other stresses. In addition, $P$. tenuiflora genes positively selected during evolution were enriched in response to temperature stimulus, response to freezing, and response to stress (Figure 3), revealing that the evolution of functional genes may also have contributed to the development of tolerance to multiple stress types, including salinity stress and chilling stress.

\section{ABA Signaling and Osmotic Adjustment Mechanisms Under Salinity Stress May Be Conserved in P. tenuiflora and Glycophytes}

According to the phylogenetic tree constructed in this study, of the 16 species included in the analysis D. glomerata was most closely related to $P$. tenuiflora (Figure 1). To our knowledge, D. glomerata is the closest sequenced species to $P$. tenuiflora. Thus, D. glomerata is an ideal plant species for use in comparative genomics and transcriptome analyses of $P$. tenuiflora and glycophytes. In this study, we compared the transcriptional responses of D. glomerata and P. tenuiflora to salinity stress. ABF is a central transcriptional factor for ABA signaling, NCED is critical gene for ABA biosynthesis, and the expression response of the LEA and dehydrin genes under salinity stress is ABAdependent (Hauser et al., 2011; Pedrosa et al., 2015; Sah et al., 2016). Our results showed that $P$. tenuiflora and $D$. glomerata both showed enhanced expression of $A B F, N C E D$, dehydrin, and $L E A$ genes under salinity stress (Figure 6B). The redundant expression of a number of dehydrin and LEA genes under salinity stress is also a common salinity tolerance characteristic for P. tenuiflora and D. glomerata (Figures 6, 7). These similar gene expression responses suggested that the ABA signaling system may be conserved in $P$. tenuiflora and glycophytes. LEA and dehydrin proteins played critical roles in osmotic adjustment and the prevention of protein aggregation under salinity or drought stress (Goyal et al., 2005). Although the response pattern of the $P$. tenuiflora LEA superfamily to salinity stress was similar to that of the glycophytes (Goyal et al., 2005; Rorat, 2006), the number of LEA5 genes in P. tenuiflora was higher than that in O. sativa, $H$. vulgare, A. tauschii, B. distachyon, and 
TABLE 1 | Puccinellia tenuiflora salinity-tolerant genes that were positively selected during evolutionary history.

\begin{tabular}{ll}
\hline Gene ID & Function description \\
\hline PtO_1679.40 & Inward rectifier potassium channel Kir2.1 \\
PtO_1088.1 & Inward rectifier potassium channel Kir2.2 \\
PtF_293.37 & Potassium channel THIK \\
PtO_1952.35 & Voltage-gated potassium channel \\
PtF_523.125 & Voltage-gated potassium channel Kv1.3 \\
PtO_1553.15 & Voltage-gated potassium channel Kv3.4 \\
PtO_1172.14 & Voltage-gated potassium channel Kv4.3 \\
PtF_19.3 & Voltage-gated potassium channel KCNQ1 \\
PtO_1180.6 & Voltage-gated potassium channel Kv1.6 \\
\hline
\end{tabular}

D. glomerata. The high number of LEA5 subfamily in $P$. tenuiflora may contribute to its strong tolerance to salinity stress and drought because the osmotic role of LEA relies on its protein abundance (i.e., there is a dosage effect). Interestingly, the number of SMP, LEA1, and LEA4 subfamilies in P. tenuiflora was much lower than that in D. glomerata (Figure 4A). We propose that different members or subfamilies of the LEA superfamily played various roles in salinity tolerance differentiation between $P$. tenuiflora and D. glomerata. How the LEA superfamily mediates salinity tolerance in $P$. tenuiflora should be further investigated in the future.

\section{$\mathrm{K}^{+} / \mathrm{Na}^{+}$Homeostasis Mechanisms of $P$. tenuiflora Are Distinct From Those of Glycophytes}

Under salinity stress, the competition between $\mathrm{Na}^{+}$and $\mathrm{K}^{+}$for binding sites on transporters, channels, or proteins decreases the accumulation of $\mathrm{K}^{+}$in plants. Similarly, a high cytosolic $\mathrm{K}^{+} / \mathrm{Na}^{+}$ ratio can decrease the binding frequency of $\mathrm{Na}^{+}$to enzymes or proteins with $\mathrm{K}^{+}$-binding sites. Therefore, high cytoplasmic $\mathrm{K}^{+} / \mathrm{Na}^{+}$ratios are salinity tolerance characteristics shared by all glycophytes and some halophytes (Zhu, 2003; Ashraf, 2004). Salinity stress increases $\mathrm{Na}^{+}$concentration and decreases $\mathrm{K}^{+}$ concentration in almost all plant species (Khan et al., 2000; Zhu, 2003; Parida and Das, 2005; Flowers and Colmer, 2008; Munns and Tester, 2008; Wang et al., 2009; Zhao et al., 2017). In the present work, root $\mathrm{K}^{+}$concentration was elevated in $P$. tenuiflora under salinity stress, but it was strongly reduced in all of the tested glycophytic Gramineae plants (Figure 5B). $P$. tenuiflora may have evolved a special $\mathrm{K}^{+}$uptake system that enables the roots to tolerate high external $\mathrm{Na}^{+}$concentrations. In $P$. tenuiflora roots, the enhanced $\mathrm{K}^{+}$concentration increased the $\mathrm{K}^{+} / \mathrm{Na}^{+}$ratio and relieved $\mathrm{Na}^{+}$damage to cells under salinity stress. Our comparative genomics analysis provided an explanation for the $\mathrm{K}^{+}$uptake characteristics of $P$. tenuiflora under salinity stress. Some $P$. tenuiflora gene families mediating $\mathrm{K}^{+}$uptake expanded during evolutionary history (Figure $2 \mathrm{C}$ and Supplementary Table 1 ). We propose that these expanded $\mathrm{K}^{+}$ uptake gene families may have contributed to the development of strong $\mathrm{K}^{+}$uptake capacity in $P$. tenuiflora under salinity stress because the functions of $\mathrm{K}^{+}$uptake genes rely on their protein abundance (dosage effect) (Figure 7). Indeed, some
$\mathrm{K}^{+}$channel or transporter genes were upregulated in roots of $P$. tenuiflora under salinity stress (Supplementary Table 5). Interestingly, several $P$. tenuiflora $\mathrm{K}^{+}$channel genes were positively selected during evolutionary history (Table $\mathbf{1}$ and Supplementary Table 3 ). We propose that the selection pressure imparted by high soil salinity may have driven the $P$. tenuiflora $\mathrm{K}^{+}$channel genes to evolve new functions that differ to those in glycophytes. This change may also have contributed to the development of the $\mathrm{K}^{+}$uptake characteristic of $P$. tenuiflora under salinity stress. Besides relieving $\mathrm{Na}^{+}$toxicity, improving the K-nutritional status also can greatly alleviate damage caused by low temperatures, iron toxicity, and drought (Cakmak, 2005). The evolution of $P$. tenuiflora $\mathrm{K}^{+}$uptake genes therefore also have benefitted these plants in adapting to these other types of environmental stress.

Puccinellia tenuiflora exhibited an extremely low $\mathrm{Na}^{+}$ concentration in the salinity-stressed shoots (Figure 5A). When the seedlings were exposed to $300 \mathrm{mM} \mathrm{NaCl}$ for $48 \mathrm{~h}$, the shoot $\mathrm{Na}^{+}$concentration of $P$. tenuiflora was only $10.7-19.8 \%$ of that in glycophytic Gramineae plants (Figure 5A). This result was consistent with that of Wang et al. (2009) who showed that $P$. tenuiflora also exhibited extremely lower shoot $\mathrm{Na}^{+}$concentrations than those of wheat. It has been reported that $P$. tenuiflora has a much stronger capacity to restrict the unidirectional $\mathrm{Na}^{+}$influx from the rhizosphere into the root than glycophytes (Wang et al., 2009). Our results provided an additional insight into the $\mathrm{Na}^{+}$tolerance of $P$. tenuiflora. $P$. tenuiflora may have an extremely strong capacity to control $\mathrm{Na}^{+}$influx from root into shoot through $\mathrm{Na}^{+}$excretion from shoot into the root and/or the restriction of unidirectional $\mathrm{Na}^{+}$ influx into the shoot. $\mathrm{Na}^{+}$excretion from the shoot into the root is a common salinity tolerance characteristic for glycophytes (Munns and Tester, 2008; Wang and Xia, 2018; Ganie et al., 2019). Although the transporter or channel gene mediating $\mathrm{Na}^{+}$ excretion has not yet been identified in P. tenuiflora, the $\mathrm{Na}^{+}$ excretion channel or transporter in this species was likely to be more permeable to $\mathrm{Na}^{+}$and/or incur a lower energy cost to that in other species. This hypothesis required verification using structural biology and electrophysiological techniques. In addition, the number of $P$. tenuiflora $H K T$ and $N H X$ families was higher than that of its relative D. glomerata (Supplementary Table 7). HKT1;3 was upregulated in the roots of $P$. tenuiflora at $48 \mathrm{~h}$ under salinity stress, but no HKT gene was upregulated in the roots of D. glomerata (Figure 6B). The HKT1 family has been demonstrated to mediate $\mathrm{Na}^{+}$excretion from shoots to roots in rice, Arabidopsis, and wheat (Munns and Tester, 2008; Wang and Xia, 2018; Ganie et al., 2019). Therefore, the P. tenuiflora HKT1;3 gene (PtO_1129.21) may be a candidate $\mathrm{Na}^{+}$excretion gene, which should be investigated in the future.

\section{Genome Evolution Appears to Contribute Development of Salinity Tolerance in $P$. tenuiflora}

Halophytes comprise remarkable plant species that can survive extremely high levels of soil salinity that would kill 99\% of other plant species (glycophytes) (Flowers and Yeo, 1995; 


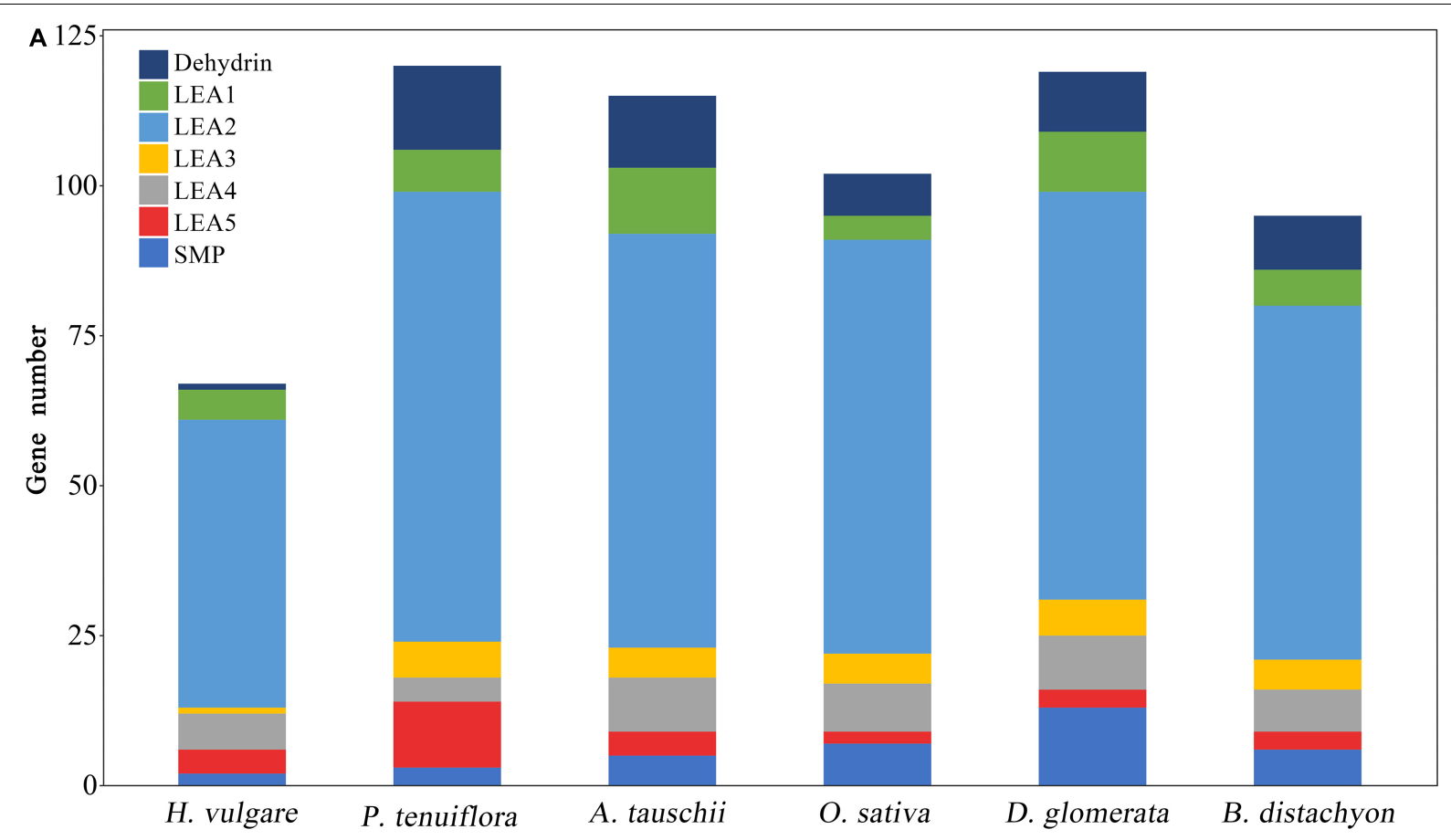

B

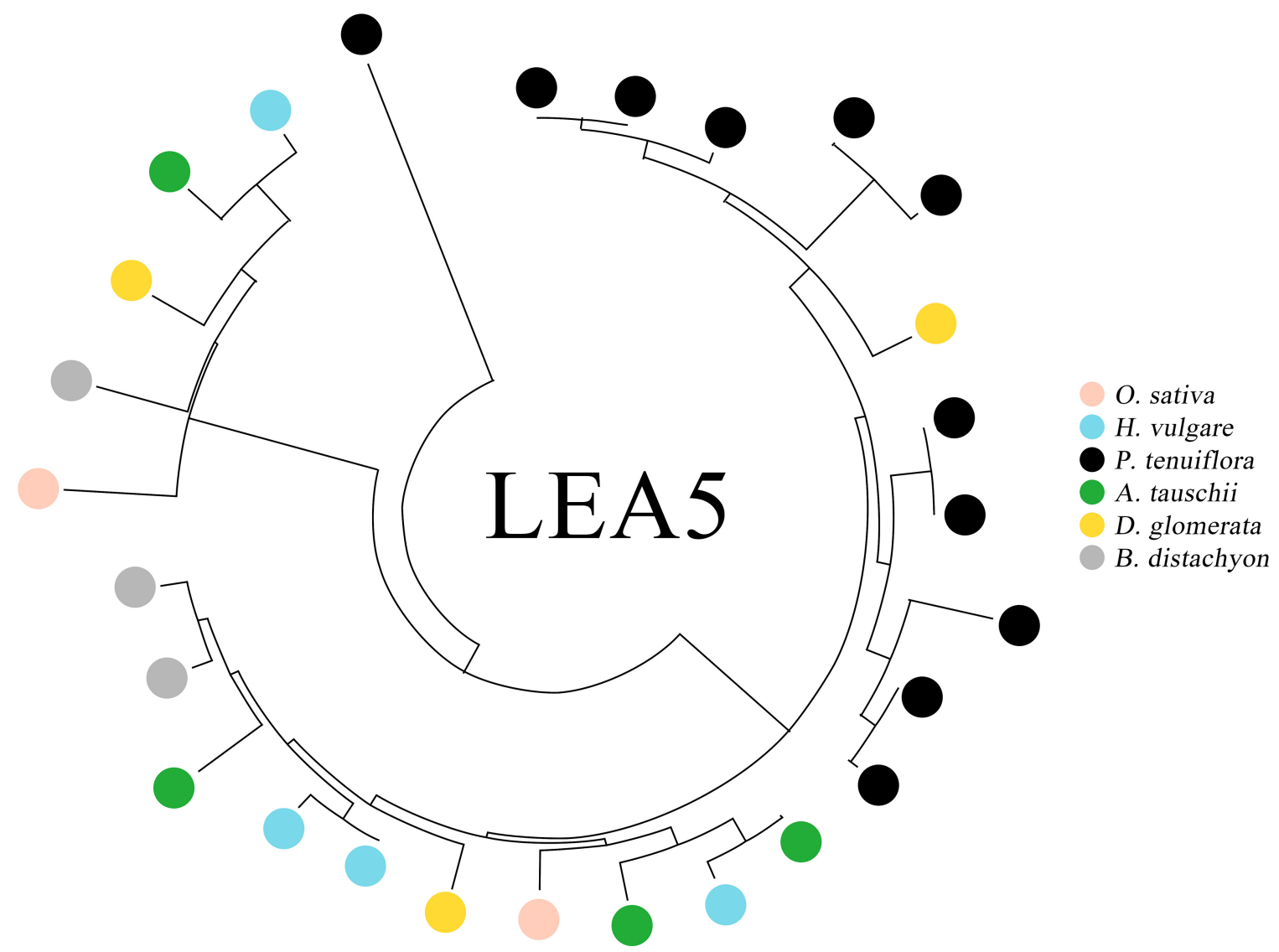

FIGURE 4 | LEA superfamily of $P$. tenuiflora. (A) Comparison of the member numbers of the LEA superfamily in $P$. tenuiflora, $D$. glomerata, rice, $H$. vulgare, B. distachyon, and A. tauschii. (B) Polygenetic tree of the LEA5 families in P. tenuiflora, D. glomerata, rice, H. vulgare, B. distachyon, and A. tauschii. Polygenetic tree constructed by MEGA-X using the Neighbor-Joining method. 


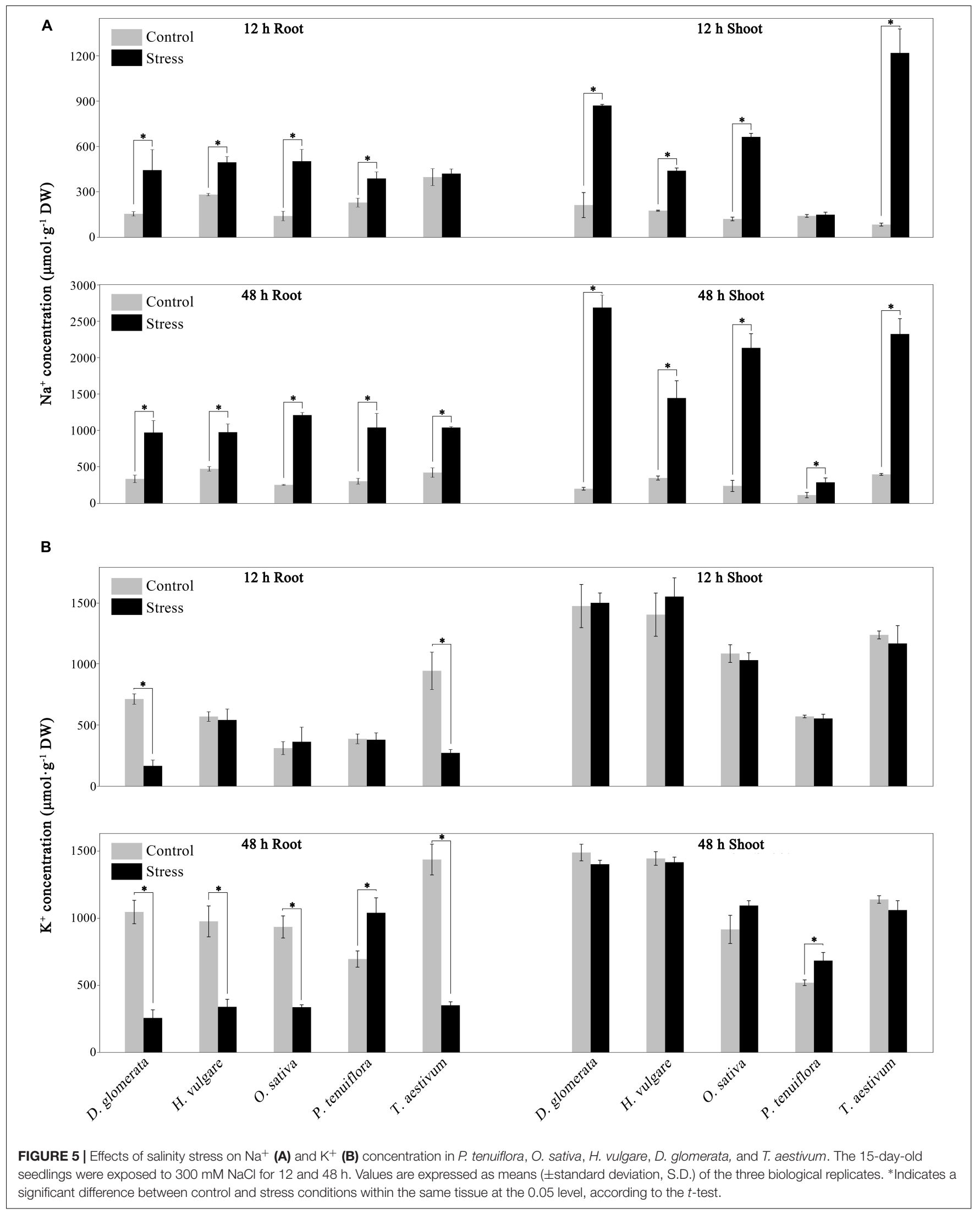



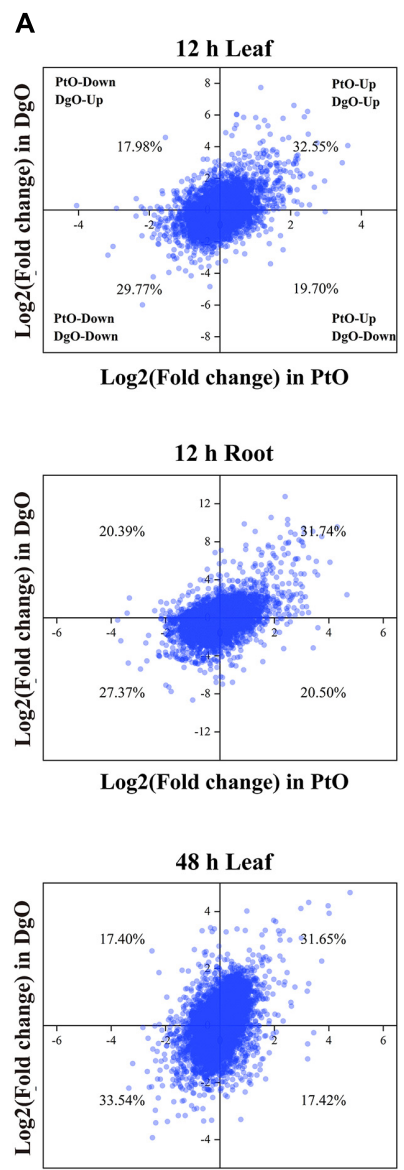

Log2(Fold change) in PtO

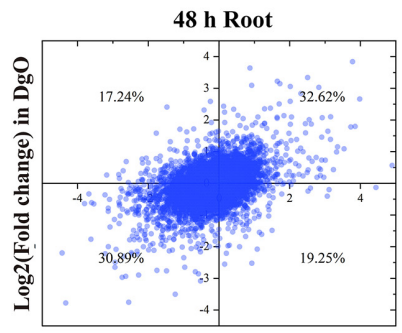

Log2(Fold change) in PtO

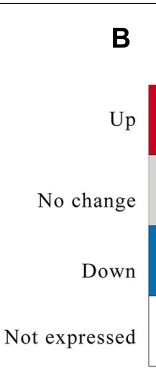

PtF 517.39

PtO_647.1

PtO_1267.15

PtF 46.10

PtF_473.89

PtO_1614.12

PtO 2103.26

PtO_1129.21

PtO__1129.5

PtF 428.24

PtF_285.12

PtF_480.27

PtF_213.113

PtF_219.42

PtF_219.43

PtO_1049.18

PtO_1336.4

PtO 2149.35

PtF 2.90_PtF 2.87

PtF_2.91

PtO_824.26

PtF 102.49

PtF_12.11

PtF 138.55

PtF_168.72

PtF_ 193.40

PtF_194.25

PtF_248.45

PtF_379.20

PtF_472.11

PtF_533.30

PtO 1196.42

PtO_1286.5

PtO 2032.66

PtO_2214.1

PtO_2527.56

PtO_661.32

$\mathrm{PtO} 701.3$

PtO_ 836.60

$\mathrm{PtO} 892.18$

PtO_921.17

PtO_576.16

PtO_1673.5

PtO_1539.26

PtF 30.90

PtF_304.68

PtF_347.43

PtO 1558.23

PtO_1784.23

PtF 144.58
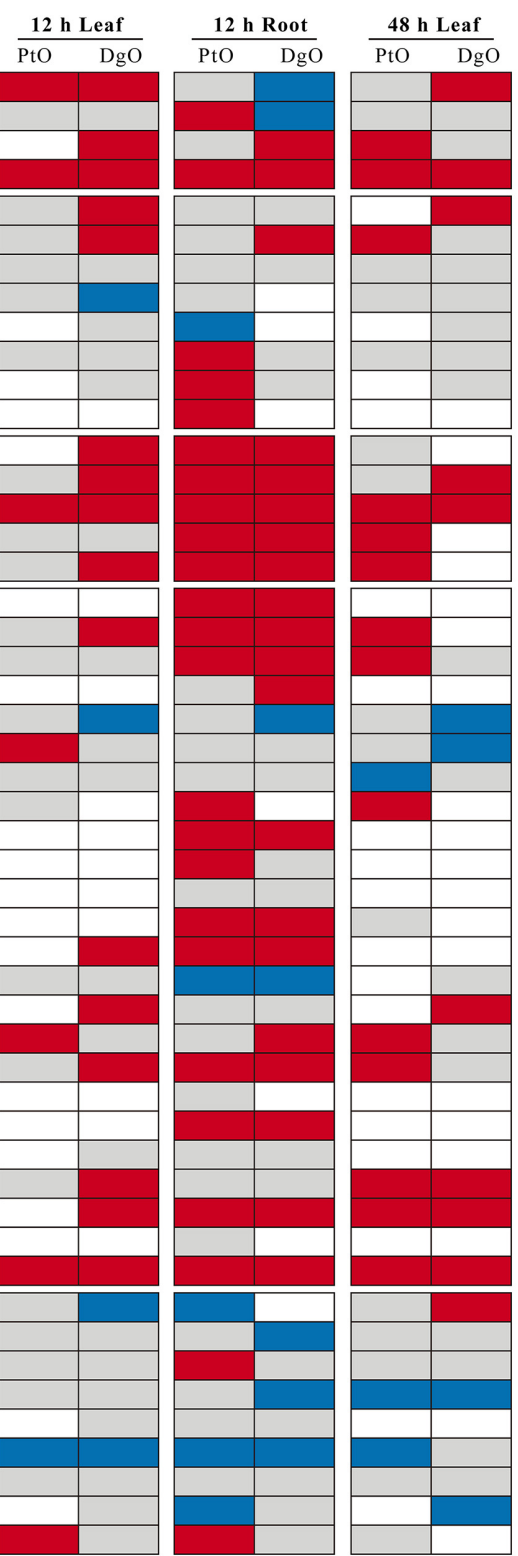

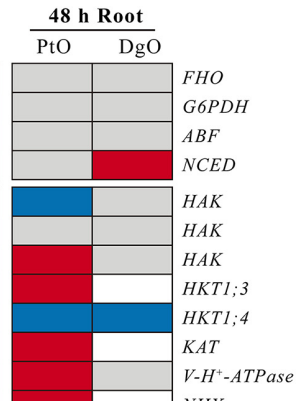

NHX
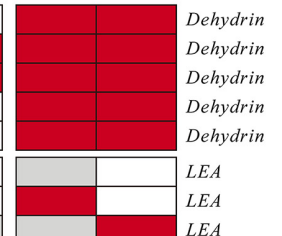
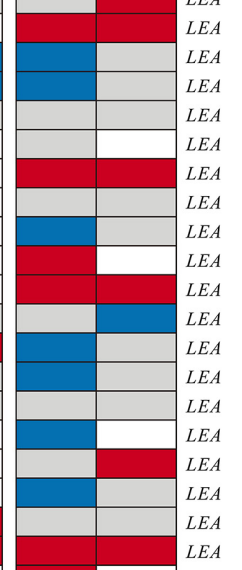

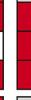

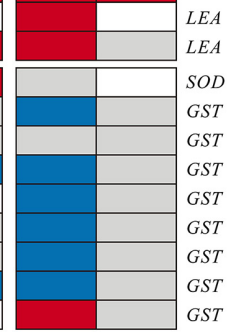

FIGURE 6 | Comparative orthologous expression response of $P$. tenuiflora and $D$. glomerata to salinity stress. (A) Rectangular coordinate system with $x=$ log 2 [fold change (stress/control) of $\mathrm{PtO}]$ and $y=\log _{2}$ (fold change of DgO) for general genes. (B) Heat map showing the expression response of the $P$. tenuiflora ortholog (PtO) and $D$. glomerata ortholog $(\mathrm{DgO})$ to salinity stress for each salinity tolerance gene. The 15-day-old seedlings were exposed to $300 \mathrm{mM}$ NaCl for 12 and $48 \mathrm{~h}$. Each treatment had three biological replicates. FHO, flavonoid 3',5'-hydroxylase; G6PDH, glucose-6-phosphate dehydrogenase; ABF, ABA responsive element binding protein; NCED, 9-cis-epoxycarotenoid dioxygenase; HAK, KUP/HAK/KT K+ transporter; HKT, high-affinity $\mathrm{K}^{+}$transporter; KAT, potassium channel KAT; LEA, late embryogenesis abundant protein; $\mathrm{NHX}, \mathrm{Na}^{+} / \mathrm{H}^{+}$exchanger; SOD, superoxide dismutase; GST, glutathione $\mathrm{S}$-transferase.

Flowers et al., 2010). It is of interest, therefore, to determine when and how halophytes achieved strong salinity tolerance and what mechanisms drive salinity tolerance differences between halophytes and glycophytes. P. tenuiflora may have inhabited saline environments for several millions of years and so may have evolved unique metabolic traits in response to its long-term exposure to severe soil salinity and drought. Our comparative genomics analysis revealed that the $P$. tenuiflora genes that were positively selected during evolutionary history were enriched in response to abiotic stimuli and response to stress (Figure 3). The selective pressure incurred by high soil salinity levels may have driven $P$. tenuiflora stress response genes to evolve new functions that differed from those of glycophytes, which may have accelerated the differentiation in salinity tolerance between P. tenuiflora and glycophytic Gramineae plants (Figure 7). In addition, expanded $P$. tenuiflora genes were found to be enriched 


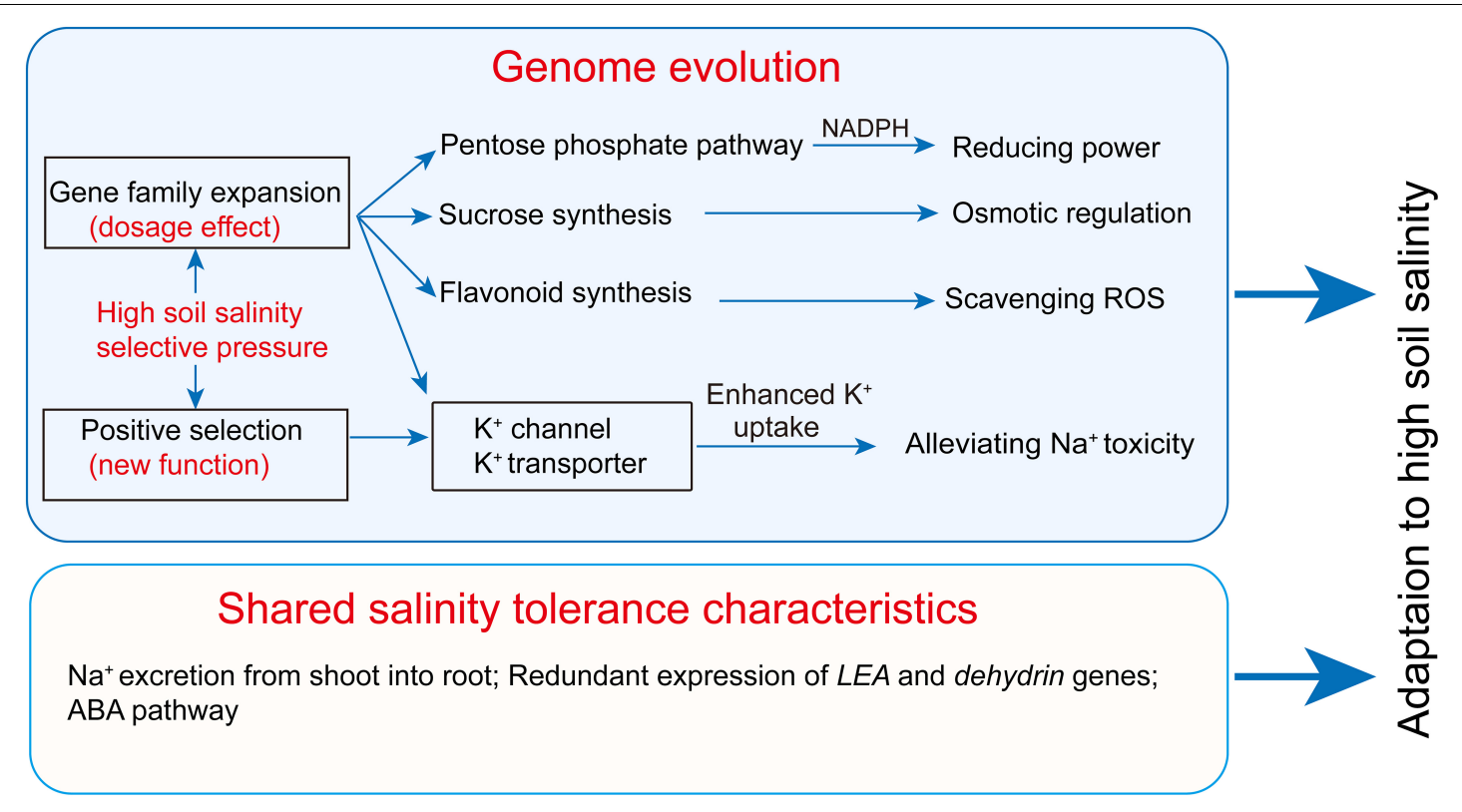

FIGURE 7 | Genomic mechanisms of salinity tolerance evolution in P. tenuiflora. LEA, late embryogenesis abundant protein; ROS, reactive oxygen species.

in the PPP, flavonoid biosynthesis, and sucrose biosynthesis (Figure 2B and Supplementary Table 2). Accordingly, a glucose6-phosphate dehydrogenase gene (a rate-controlling gene in the PPP), two flavonoid 3',5'-hydroxylase genes (critical genes for flavonoid biosynthesis), and three sucrose synthase genes were upregulated in the roots or leaves of $P$. tenuiflora under salinity stress (Supplementary Table 5). The PPP is important when plants are exposed to stress because some signals trigger the rapid diversion of $\mathrm{NADPH}$ to use in stress responses (Kruger and Schaewen, 2003; Esposito, 2016). The enhanced PPP might provide additional energy and carbon resources to fuel salinity stress responses in $P$. tenuiflora (Figure 7). Flavonoid plays important roles in the detoxification of reactive oxygen species under salinity stress (Agati et al., 2012), and sucrose is a central compatible solute for osmotic regulation (Parida and Das, 2005; Xiao et al., 2020). Therefore, the expansion and enhanced expression of flavonoid biosynthesis and sucrose biosynthesis genes in $P$. tenuiflora protected these plants from the toxicity associated with reactive oxygen species and $\mathrm{Na}^{+}$under salinity stress (Figure 7). In addition, gene families involved in the plant-pathogen interaction pathway were expended during the evolutionary history of $P$. tenuiflora (Figure 2B), which may have contributed to the development of biotic stress tolerance of P. tenuiflora.

In conclusion, the strong salinity tolerance of $P$. tenuiflora was linked to its $\mathrm{K}^{+}$uptake characteristics and strong capacity to control $\mathrm{Na}^{+}$influx into shoots, and some $P$. tenuiflora $\mathrm{K}^{+}$uptake gene families experienced expansion and positive selection during evolutionary history. During the adaptation of $P$. tenuiflora to high soil salinity, expanded salinity-tolerant genes have exerted dosage effects, and positively-selected salinitytolerant genes may have been involved in the construction of new salinity tolerance pathways. This was the genomic basis for salinity tolerance differentiation between $P$. tenuiflora and glycophytic Gramineae plants (Figure 7).

\section{DATA AVAILABILITY STATEMENT}

The original contributions generated for this study are publicly available. This data can be found here: All raw data of transcriptional analysis are available at NCBI (accession number PRJNA673532/SRR12957599-SRR12957646). The genome sequence of $P$. tenuiflora is available from NCBI (https://www. ncbi.nlm.nih.gov/genome/?term=QRDG00000000.1) (accession number QRDG00000000.1).

\section{AUTHOR CONTRIBUTIONS}

CY and RG: experiment design. RG, LZ, KZ, HL, NB, and CY: performance of experiments. RG, CY, LZ, and KZ: data analysis. CY, RG, LZ, and KZ: manuscript writing. All authors read and approved the final manuscript.

\section{FUNDING}

This work was supported by the National Natural Science Foundation of China (No. 31971762) and the Fundamental Research Funds for the Central Universities (No. 2412019FZ026). The funding bodies played no role in the design of the study and collection, analysis, and interpretation of data and in writing the manuscript. 


\section{SUPPLEMENTARY MATERIAL}

The Supplementary Material for this article can be found online at: https://www.frontiersin.org/articles/10.3389/fpls.2021. 649001/full\#supplementary-material

Supplementary Figure 1 | Gene number in Puccinellia tenuiflora and several sequenced Gramineae plants.

Supplementary Figure 2 | Motif component comparison of $P$. tenuiflora, Dactylis glomerata, rice, Hordeum vulgare, and Aegilops tauschii for each $\mathrm{NHX}$ protein.

Supplementary Figure $\mathbf{3}$ | Motif component comparison of $P$. tenuiflora, D. glomerata, rice, $H$. vulgare, and $A$. tauschii for each HKT protein.

Supplementary Figure 4 | Motif component comparison of $P$. tenuiflora, D. glomerata, rice, H. vulgare, and A. tauschii for each AKT protein.

Supplementary Figure 5 | Effects of salinity stress on the $\mathrm{Na}^{+} / \mathrm{K}^{+}$ratio in P. tenuiflora, O. sativa, H. vulgare, D. glomerata, and T. aestivum. The 15-day-old seedlings were exposed to $300 \mathrm{mM} \mathrm{NaCl}$ for $12 \mathrm{~h}$ and $48 \mathrm{~h}$. Values are expressed as means ( \pm standard deviation, S.D.) of the three biological replicates. * indicates significant difference between control and stress conditions within the same tissue at the 0.05 level, according to the $t$-test.

\section{REFERENCES}

Agati, G., Azzarello, E., Pollastri, S., and Tattini, M. (2012). Flavonoids as antioxidants in plants: location and functional significance. Plant Sci. 196 67-76. doi: 10.1016/j.plantsci.2012.07.014

Ardie, S. W., Liu, S. K., and Takano, T. (2010). Expression of the AKT1-type K+ channel gene from Puccinellia tenuiflora, PutAKT1, enhances salt tolerance in Arabidopsis. Plant Cell Rep. 29, 865-874. doi: 10.1007/s00299-010-0872-2

Ardie, S. W., Nishiuchi, S., Liu, S. K., and Takano, T. (2011). Ectopic expression of the channel $\beta$ subunits from Puccinellia tenuiflora (KPutB1) and rice (KOB1) alters $\mathrm{K}+/ \mathrm{Na}+$ homeostasis of yeast and Arabidopsis. Mol. Biotechnol. 48, 76-86. doi: 10.1007/s12033-010-9349-3

Ardie, S. W., Xie, L., Takahashi, R., Liu, S. K., and Takano, T. (2009). Cloning of a high-affinity $\mathrm{K}+$ transporter gene PutHKT2;1 from Puccinellia tenuiflora and its functional comparison with OsHKT2; 1 from rice in yeast and Arabidopsis. J. Exp. Bot. 60, 3491-3502.

Ashraf, M. (2004). Some important physiological selection criteria for salt tolerance in plants. Flora 199, 361-376. doi: 10.1078/0367-2530-00165

Bailey, T. L., Bodén, M., Buske, F. A., Frith, M., Grant, C. E., Clementi, L., et al. (2009). MEME SUITE: tools for motif discovery and searching. Nucleic Acids Res. 37, 202-208.

Cai, J., Liu, X., Vanneste, K., Proost, S., Tsai, W. C., Liu, K. W., et al. (2015). The genome sequence of the orchid Phalaenopsis equestris. Nat. Genet. 47, 65-72.

Cakmak, I. (2005). The role of potassium in alleviating detrimental effects of abiotic stresses in plants. J. Soil Sci. Plant Nutr. 168, 521-530. doi: 10.1002/ jpln.200420485

Castresana, J. (2000). Selection of conserved blocks from multiple alignments for their use in phylogenetic analysis. Mol. Biol. Evol. 17, 540-552. doi: 10.1093/ oxfordjournals.molbev.a026334

De Bie, T., Demuth, J. P., Cristianini, N., and Hahn, M. W. (2006). CAFE: a computational tool for the study of gene family evolution. Bioinformatics 22, 1269-1271. doi: 10.1093/bioinformatics/btl097

Edgar, R. C. (2004). MUSCLE: multiple sequence alignment with high accuracy and high throughput. Nucleic Acids Res. 32, 1792-1797. doi: 10.1093/nar/gkh340

Esposito, S. (2016). Nitrogen assimilation, abiotic stress and glucose 6-phosphate dehydrogenase: the full circle of reductants. Plants 5, 236-246.

Finn, R. D., Attwood, T. K., Babbitt, P. C., Bateman, A., Bork, P., Bridge, A. J., et al. (2017). InterPro in 2017-beyond protein family and domain annotations. Nucleic Acids Res. 45, D190-D199.
Supplementary Figure 6 | KEGG enrichment of DEGs in P. tenuiflora under salinity stress. The 15-day-old seedlings were exposed to $300 \mathrm{mM} \mathrm{NaCl}$ for 12 and $48 \mathrm{~h}$. Each treatment had three biological replicates.

Supplementary Table 1 | Members of the $P$. tenuiflora gene families that expanded during evolutionary history.

Supplementary Table 2 | Enriched GO terms in the GO enrichment analysis of the $P$. tenuiflora gene families that expanded during evolutionary history.

Supplementary Table 3 | List of $P$. tenuiflora genes that were positively selected during evolutionary history.

Supplementary Table 4 | Differentially expressed genes in P. tenuiflora under salinity stress. The 15-day-old seedlings were exposed to $300 \mathrm{mM} \mathrm{NaCl}$ for 12 and $48 \mathrm{~h}$. Each treatment had three biological replicates.

Supplementary Table 5 | Differentially expressed salinity-tolerant genes in $P$. tenuiflora under salinity stress. The 15-day-old seedlings were exposed to $300 \mathrm{mM} \mathrm{NaCl}$ for 12 and $48 \mathrm{~h}$. Each treatment had three biological replicates.

Supplementary Table 6 | Comparative fold changes (stress/control) of qRT-PCR and RNA-seq in P. tenuiflora roots at $48 \mathrm{~h}$. Each treatment had three biological replicates, and each biological replicate comprised a pool of 10 plants. The gene-specific primers were displayed.

Supplementary Table 7 | Number of HKT, AKT, and NHX families in P. tenuiflora, rice, D. glomerata, and Arabidopsis.

Flowers, T. J., and Colmer, T. D. (2008). Salinity tolerance in halophytes. New Phytol. 179, 945-963. doi: 10.1111/j.1469-8137.2008.02531.x

Flowers, T. J., Galal, H. K., and Bromham, L. (2010). Evolution of halophytes: multiple origins of salt tolerance in land plants. Funct. Plant Biol. 37, 604-612. doi: 10.1071/fp09269

Flowers, T. J., and Yeo, A. R. (1995). Breeding for salinity resistance in crop plants where next ? Funct. Plant Biol. 22, 875-884. doi: 10.1071/pp9950875

Ganie, S. A., Molla, K. A., Henry, R. J., Bhat, K. V., and Mondal, T. K. (2019). Advances in understanding salt tolerance in rice. Theor. Appl. Genet. 132, 851-870. doi: 10.1007/s00122-019-03301-8

Goyal, K., Walton, L. J., and Tunnacliffe, A. (2005). LEA proteins prevent protein aggregation due to water stress. Biochem. J. 388, 151-157. doi: 10.1042/ bj20041931

Guo, R., Zhao, L., Zhang, K., Gao, D., and Yang, C. (2020). Genome of extreme halophyte Puccinellia tenuiflora. BMC Genomics 21:311. doi: 10.1186/s12864020-6727-5

Han, M. V., Thomas, G. W. C., Lugo-Martinez, J., and Hahn, M. W. (2013). Estimating gene gain and loss rates in the presence of error in genome assembly and annotation using CAFE 3. Mol. Biol. Evol. 30, 1987-1997. doi: 10.1093/ molbev/mst100

Hauser, F., Waadt, R., and Schroeder, J. (2011). Evolution of abscisic acid synthesis and signaling mechanisms. Curr. Biol. 21, R346-R355.

Huang, L., Feng, G., Yan, H., Zhang, Z., Bushman, S. B., Wang, J., et al. (2020). Genome assembly provides insights into the genome evolution and flowering regulation of orchardgrass. Plant Biotechnol. J. 18, 373-388. doi: 10.1111/pbi. 13205

Khan, M. A., Ungar, I. A., and Showalter, A. M. (2000). The effect of salinity on the growth, water status, and ion content of a leaf succulent perennial halophyte, Suaeda fruticosa (L.) Forssk. J. Arid Environ. 45, 73-84. doi: 10.1006/jare.1999. 0617

Kruger, N. J., and Schaewen, A. (2003). The oxidative pentose phosphate pathway: structure and organisation. Curr. Opin. Plant Biol. 6, 236-246. doi: 10.1016/ s1369-5266(03)00039-6

Kumar, S., Stecher, G., Li, M., Knyaz, C., and Tamura, K. (2018). MEGA X: molecular evolutionary genetics analysis across computing platforms. Mol. Biol. Evol. 35, 1547-1549. doi: 10.1093/molbev/msy096

Lambeck, K., Rouby, H., Purcell, A., Sun, Y., and Sambridge, M. (2014). Sea level and global ice volumes from the last glacial maximum to the holocene. Proc. Natl. Acad. Sci. U.S.A. 111, 15296-15303. doi: 10.1073/pnas.1411762111 
Li, L., Stoeckert, C. J. J., and Roos, D. S. (2003). OrthoMCL: identification of ortholog groups for eukaryotic genomes. Genome Res. 13, 2178-2189. doi: 10.1101/gr.1224503

Liu, H., Zhang, X. X., Takano, T., and Liu, S. K. (2009). Characterization of a PutCAX1 gene from Puccinellia tenuiflora that confers $\mathrm{Ca} 2+$ and $\mathrm{Ba} 2+$ tolerance in yeast. Biochem. Bioph. Res. Co. 383, 392-396. doi: 10.1016/j.bbrc. 2009.04.042

Livak, K. J., and Schmittgen, T. D. (2001). Analysis of relative gene expression data using real-time quantitative PCR and the 2(-Delta Delta C(T)) method. Methods 25, 402-408. doi: 10.1006/meth.2001.1262

Ma, J., and Bennet-zen, J. L. (2004). Rapid recent growth and divergence of rice nuclear genomes. Proc. Natl. Acad. Sci. U.S.A. 101, 12404-12410. doi: 10.1073/ pnas.0403715101

Mao, X., Cai, T., Olyarchuk, J. G., and Wei, L. (2005). Automated genome annotation and pathway identification using the KEGG Orthology (KO) as a controlled vocabulary. Bioinformatics 21, 3787-3793. doi: 10.1093/ bioinformatics/bti430

Meng, X., Zhao, Q., Jin, Y., Yu, J., Yin, Z., Chen, S., et al. (2016). Chillingresponsive mechanisms in halophyte Puccinellia tenuiflora seedlings revealed from proteomics analysis. J. Proteomics 143, 365-381. doi: 10.1016/j.jprot.2016. 04.038

Munns, R., and Tester, M. (2008). Mechanisms of salinity tolerance. Annu. Rev. Plant Biol. 59, 651-681. doi: 10.1146/annurev.arplant.59.032607.092911

Parida, A. K., and Das, A. B. (2005). Salt tolerance and salinity effects on plants: a review. Ecotox. Environ. Safe. 60, 324-349. doi: 10.1016/j.ecoenv.2004.06.010

Pedrosa, A. M., Martins, C. P. S., Gonçalves, L. P., and Costa, M. G. C. (2015). Late embryogenesis abundant (LEA) constitutes a large and diverse family of proteins involved in development and abiotic stress responses in sweet Orange (Citrus sinensis L. Osb.). PLoS One 10:e0145785. doi: 10.1371/journal.pone. 0145785

Rorat, T. (2006). Plant dehydrins-tissue location, structure and function. Cell Mol. Biol. Lett. 11, 536-556.

Sah, S. K., Reddy, K. R., and Li, J. (2016). Abscisic acid and abiotic stress tolerance in crop plants. Front. Plant Sci. 7:571. doi: 10.3389/fpls.2016.00571

Stamatakis, A. (2006). RAxML-VI-HPC: maximum likelihood-based phylogenetic analyses with thousands of taxa and mixed models. Bioinformatics 22, 26882690. doi: 10.1093/bioinformatics/btl446

Stamatakis, A., Hoover, P., and Rougemont, J. A. (2008). Rapid bootstrap algorithm for the RAxML web servers. Syst. Biol. 57, 758-771. doi: 10.1080/ 10635150802429642

Suzuki, K., Yamaji, N., Costa, A., Okuma, E., Kobayashi, N. I., Kashiwagi, T., et al. (2016). OsHKT1;4-mediated Na+ transport in stems contributes to $\mathrm{Na}+$ exclusion from leaf blades of rice at the reproductive growth stage upon salt stress. BMC Plant Biol. 16:22. doi: 10.1186/s12870-016-0709-4

Wang, C., Zhang, J., Liu, X., Li, Z., Wu, G., Cai, J., et al. (2009). Puccinellia tenuiflora maintains a low $\mathrm{Na}+$ level under salinity by limiting unidirectional $\mathrm{Na}+$ influx resulting in a high selectivity for $\mathrm{K}+$ over Na+. Plant Cell Environ. 32, 486-496. doi: 10.1111/j.1365-3040.2009.01942.x

Wang, F., Chen, Z. H., Liu, X., Salih, A., and Shabala, S. (2019). The loss of RBOHD function modulates root adaptive responses to combined hypoxia and salinity stress in Arabidopsis. Environ. Exp. Bot. 158, 125-135. doi: 10.1016/j.envexpbot. 2018.11.020

Wang, M., and Xia, G. (2018). The landscape of molecular mechanisms for salt tolerance in wheat. Crop J. 6, 42-47. doi: 10.1016/j.cj.2017.09.002

Wang, X., Sun, G., Wang, J., Cao, W., Liang, J., Yu, Z., et al. (2006). Relationships among MDA content, plasma membrane permeability and the chlorophyll fluorescence parameters of Puccinellia tenuiflora seedlings under $\mathrm{NaCl}$ stress. Acta Ecol. Sin. 26, 122-129.

Wang, Y. C., Chu, Y., Liu, G., Wang, M. H., Jiang, J., Hou, Y., et al. (2007a). Identification of expressed sequence tags in an alkali grass (Puccinellia tenuiflora) cDNA library. J. Plant Physiol. 164, 78-89. doi: 10.1016/j.jplph.2005. 12.006

Wang, Y. C., Yang, C. P., Liu, G. F., and Jiang, J. (2007b). Development of a cDNA microarray to identify gene expression of Puccinellia tenuiflora under salinealkali stress. Plant Physiol. Biochem. 45, 567-576. doi: 10.1016/j.plaphy.2007. 05.006
Xiao, B., Lu, H., Li, C., Bhanbhro, N., Cui, X., and Yang, C. (2020). Carbohydrate and plant hormone regulate the alkali stress response of hexaploid wheat (Triticum aestivum L.). Environ. Exp. Bot. 175, 104053. doi: 10.1016/j. envexpbot.2020.104053

Xu, A. (1990). Application of Puccinellia chinampoensis and Puccinellia tenuiflora in Western Jilin Province of China. China Grassl. 2, 62-65.

Xu, H., Bao, C., Ge, C., and Li, P. (1995). Comparative study of salt tolerance of two salt-tolerant herbages Puccinellia chinampoensis and Puccinellia tenuiflora. China Grassl. 14, 43-47.

Yan, X. F., and Sun, G. R. (2000). Physiological Ecology Research of Puccinellia tenuiflora. Beijing: Science Press.

Yang, Z. (2007). PAML 4: phylogenetic analysis by maximum likelihood. Mol. Biol. Evol. 24, 1586-1591. doi: 10.1093/molbev/msm088

Young, M. D., Wakefield, M. J., Smyth, G. K., and Oshlack, A. (2010). Gene ontology analysis for RNA-seq: accounting for selection bias. Genome Biol. 11, r14. doi: 10.1186/gb-2010-11-2-r14

Yu, J. J., Chen, S. X., Wang, T., Sun, G. R., and Dai, S. J. (2013). Comparative proteomic analysis of Puccinellia tenuiflora leaves under $\mathrm{Na} 2 \mathrm{CO} 3$ stress. Int. J. Mol. Sci. 14, 1740-1762. doi: 10.3390/ijms14011740

Yu, J. J., Chen, S. X., Zhao, Q., Wang, T., Yang, C. P., Diaz, C., et al. (2011). Physiological and proteomic analysis of salinity tolerance in Puccinellia tenuiflora. J. Proteome Res. 10, 3852-3870. doi: 10.1021/pr101 $102 \mathrm{p}$

Yuan, Y., Jin, X., Liu, J., Zhao, X., Zhou, J., Wang, X., et al. (2018). The Gastrodia elata genome provides insights into plant adaptation to heterotrophy. Nat. Commun. 9:1615.

Zhang, J., Nielsen, R., and Yang, Z. (2005). Evaluation of an improved branch-site likelihood method for detecting positive selection at the molecular level. Mol. Biol. Evol. 22, 2472-2479. doi: 10.1093/molbev/msi237

Zhang, W. D., Wang, P., Bao, Z., Ma, Q., Duan, L. J., Bao, A. K., et al. (2017). SOS1, HKT1;5, and NHX1 synergistically modulate $\mathrm{Na}+$ homeostasis in the halophytic grass Puccinellia tenuiflora. Front. Plant Sci. 8:576. doi: 10.3389/fpls. 2017.00576

Zhang, X., Wei, L., Wang, Z., and Wang, T. (2013). Physiological and molecular features of Puccinellia tenuiflora tolerating salt and alkaline-salt stress. J. Integr. Plant Biol. 55, 262-276. doi: 10.1111/jipb.12013

Zhao, K., Song, J., Feng, G., Zhao, M., and Liu, J. (2011). Species, types, distribution, and economic potential of halophytes in China. Plant Soil 342, 495-509. doi: 10.1007/s11104-010-0470-7

Zhao, L., Yang, Z., Guo, Q., Mao, S., Sun, F., Wang, H., et al. (2017). Transcriptomic profiling and physiological responses of halophyte kochia sieversiana provide insights into salt tolerance. Front. Plant Sci. 8:1985. doi: 10.3389/fpls.2017. 01985

Zhao, Q., Suo, J., Chen, S., Jin, Y., Yin, Z., Zhang, Y., et al. (2016). Chillingresponsive mechanisms in halophyte Puccinellia tenuiflora seedlings revealed from proteomics analysis. Sci. Rep. U.K. 6:32717.

Zhu, D., Hou, L., Xiao, P., Guo, Y., Deyholos, M. K., and Liu, X. (2019). VvWRKY30, a grape WRKY transcription factor, plays a positive regulatory role under salinity stress. Plant Sci. 280, 132-142. doi: 10.1016/j.plantsci.2018.03. 018

Zhu, J. K. (2003). Regulation of ion homeostasis under salt stress. Curr. Opin. Plant Biol. 6, 441-445. doi: 10.1016/s1369-5266(03)00085-2

Conflict of Interest: KZ was employed by Beijing Novogene Bioinformatics Technology Ltd.

The remaining authors declare that the research was conducted in the absence of any commercial or financial relationships that could be construed as a potential conflict of interest.

Copyright $\odot 2021$ Guo, Zhao, Zhang, Lu, Bhanbhro and Yang. This is an open-access article distributed under the terms of the Creative Commons Attribution License (CC BY). The use, distribution or reproduction in other forums is permitted, provided the original author(s) and the copyright owner(s) are credited and that the original publication in this journal is cited, in accordance with accepted academic practice. No use, distribution or reproduction is permitted which does not comply with these terms. 